High Energy Phenomena in Relativistic Outflows III (HEPRO III)

International Journal of Modern Physics: Conference Series

Vol. 8 (2012) 31-36

(C) World Scientific Publishing Company

DOI: $10.1142 / \mathrm{S} 2010194512004382$

\title{
HARD GAMMA-RAY SOURCE SPECTRA IN TeV BLAZARS
}

\author{
EVA LEFA \\ Max-Planck-Institut für Kernphysik, P.O. Box 103980, 69029 Heidelberg, Germany \\ Landessternwarte, Königstuhl 12, 69117 Heidelberg, Germany \\ eva.lefa@mpi-hd.mpg.de \\ FRANK M. RIEGER \\ Max-Planck-Institut für Kernphysik, P.O. Box 103980, 69029 Heidelberg, Germany \\ European Associated Laboratory for Gamma-Ray Astronomy, jointly supported by \\ $C N R S$ and $M P G$ \\ Frank.Rieger@mpi-hd.mpg.de \\ FELIX A. AHARONIAN \\ Max-Planck-Institut für Kernphysik, P.O. Box 103980, 69029 Heidelberg, Germany \\ Dublin Institute for Advanced Studies, 31 Fitzwilliam Place, Dublin 2, Ireland \\ Felix.Aharonian@mpi-hd.mpg.de
}

\begin{abstract}
The problem of the hard (intrinsic) $\gamma$-ray source spectra in $\mathrm{TeV}$ blazars is examined. We show that relativistic Maxwell-like particle distributions provide a suitable interpretation for the inferred hard, very high energy (VHE) photon spectra. We discuss the potential of these distributions to produce broader and softer spectra if multiple zones contribute to the observed emission. We show that the dominance of one component could lead to a VHE flaring state with hard spectral features, like the one observed for Mkn 501 in July 2009.
\end{abstract}

Keywords: Blazars; Gamma-ray emission; Stochastic acceleration; Maxwellian distribution.

\section{Introduction}

Over the last few years the origin of the very hard, intrinsic $\gamma$-ray source spectra in blazars has been intensively discussed. Blazars can produce $\mathrm{TeV}$ radiation, and this radiation can interact with photons of the Extragalactic Background Light (EBL) as it travels over cosmological distances to reach the observer, via the process $\gamma \gamma_{\mathrm{EBL}} \rightarrow e^{+} e^{-}$(see Ref. 1). Thus, although the observed TeV spectra of these sources often appear very steep, their de-absorbed (EBL-corrected) spectra can be intrinsically hard. The level of attenuation depends strongly on the EBL flux level and spectrum, and current uncertainties on the EBL (see Ref. 2 for a recent review) introduce some difficulties in defining how hard the absorption-corrected source spectra are. 
However, in some characteristic cases, like for the distant blazars 1ES 1101-232 $(z=0.186)$ and 1ES $0229+200(z=0.139)$, the emitted TeV spectra still tend to be very hard, with intrinsic photon indices $\Gamma \leq 1.5$, even when corrected for low EBL flux levels. ${ }^{3,4}$

The recent Fermi detection of variable $\gamma$-ray emission from the nearby $(z=$ 0.034) TeV blazar Mkn 501 in $2009^{5}$ has provided strong additional evidence for the presence of hard intrinsic $\gamma$-ray source spectra independently of questions related to the EBL. The original Fermi-paper on Mkn 501 already suggested that the spectrum above $10 \mathrm{GeV}$ became much harder during a ( 30-day) flaring state in $2009 .{ }^{5} \mathrm{An}$ independent analysis of the same data by Ref. 6 reported that the flare spectrum $(10 \mathrm{GeV}-200 \mathrm{GeV})$ could possibly be as hard as $\Gamma \simeq 1.1$.

The existence of such hard intrinsic VHE spectra poses strong challenges to conventional interpretation based on leptonic Synchrotron self-Compton (SSC) and External Compton (EC) models. This is related to the fact that any hard injection spectrum of electrons, even a monochromatic one, is normally expected to quickly undergo radiative (synchrotron or Thomson) cooling and thereby develop a standard $E_{e}^{-2}$-form with corresponding IC (Thomson) $\gamma$-ray photon spectrum $E_{\gamma}^{-1.5}$. In addition, the suppression of the cross-section due to Klein-Nishina (KN) effects usually leads to even steeper spectra $(\Gamma>1.5)$ at $\mathrm{TeV}$ energies.

A number of alternative explanations have been explored in the literature to overcome this problem (see, e.g., Refs. 7-15). In the context of leptonic models we know, for example, that narrow, energetic electron distributions are able to reproduce hard $\gamma$-ray source spectra provided radiative losses do not modify the electron distribution This is the case for a power-law electron distribution with a large value of the minimum cut-off, provided the magnetic field is sufficiently small to avoid radiative losses, ${ }^{16}$ or provided adiabatic losses dominate ${ }^{17}$.

A possible way to take radiative looses explicitly into account, however, is to consider relativistic Maxwell-like electron distributions that can be formed by a stochastic acceleration processes which is balanced by synchrotron or Thomson losses. ${ }^{17}$. These particle distributions are especially attractive for the interpretation of the inferred hard $\gamma$-ray source spectra because they resemble, to some extent, mono-energetic distributions (i.e., the hardest possible particle injection spectra).

Below we show that in a standard, one-zone SSC model, VHE spectra as hard as $E_{\gamma} d N / d E_{\gamma} \propto E_{\gamma}^{1 / 3}$ can be reproduced (see Ref. 17 for more details). We also discuss the potential of such distributions to form broader and softer emission spectra in ("multi-zone") cases where the observed radiation comes from more than one emitting region ("blob"). In this situation, distinct hard VHE spectral features can appear once a "leading" zone becomes dominant over the emission from the other components (see Ref. 18 for more details). Below this is illustrated for the noted flare in Mkn 501. 


\section{Maxwellian-Like Electron Distributions and Hard VHE Spectra}

Relativistic Maxwellian-like electron distributions can be the outcome of a stochastic acceleration process (e.g., 2nd order Fermi) that is balanced by synchrotron (and/or Compton) energy losses, or in general any energy loss mechanism that exhibits a quadratic dependence on the particle energy ${ }^{19,20}$. Electrons are then accelerated by, e.g., scattering off randomly moving Alfaén waves in an isotropic turbulent medium up to a maximum characteristic energy $\gamma_{c}$, the "temperature" of the distribution, at which acceleration and cooling time scales become equal. For synchrotron losses

$$
\frac{d p}{d t}=-\beta_{s} p^{2}=-\frac{4}{3}\left(\sigma_{T} / m_{e}^{2} c^{2}\right) U_{B} p^{2}
$$

where $p$ is the particle momentum and $U_{B}$ the energy density of the magnetic field, this "temperature" $\gamma_{c}$ is

$$
\gamma_{c}=\left(\frac{b_{1} D_{0}}{\beta_{s}}\right)^{1 / b_{1}}\left(m_{e} c\right)^{-1}
$$

Here, $D_{0}$ is given by the diffusion coefficient in momentum space $D_{p}=\frac{p^{2}}{3 \tau}\left(\frac{V_{A}}{c}\right)^{2} \equiv$ $D_{0} p^{3-b_{1}}$, with $V_{A}=\frac{B}{\sqrt{4 \pi \rho}}$ the Alfvén speed defined by the magnetic field $B$ and the bulk flow density $\rho$. $\tau$ is the mean scattering time, $\tau=\lambda / c \propto p^{b_{1}-1}, b_{1} \geq 1$. The factor $b_{1}$ is related to the considered turbulent wave spectrum $W(k) \propto k^{-q}$ via $b_{1}=$ $3-q$. The steady-state solution of the corresponding Fokker-Planck equation that describes the aforementioned situation is a relativistic Maxwellian-like distribution

$$
n(\gamma)=A \gamma^{2} e^{-\left(\frac{\gamma}{\gamma_{c}}\right)^{b_{1}}}
$$

with constant $A$ to be defined by the relevant initial conditions. These distributions are very narrow and resemble, to some extent, the behavior of a $\delta$-function. The synchrotron spectrum that arises is dominated by the emission of electrons with $\gamma_{c}$. It exhibits the characteristic 1/3-slope up to the corresponding "synchrotron cut-off energy" $h \nu_{c}^{\text {syn }} \sim \delta b \gamma_{c}^{2}$ where $b=B / B_{c r}$ and $B_{c r}=m^{2} c^{3} / e \hbar$. The same holds for the Compton spectrum. The 1/3-slope appears up to the "Compton cut-off energy", reflecting the synchrotron spectrum. Depending on whether we are in the KN or Thomson regime, the observed Compton cut-off frequency corresponds to $\delta \gamma_{c} m c^{2}$ (if $b \gamma_{c}^{3}>1$ ) or to $\delta b \gamma_{c}^{4}$ (if $b \gamma_{c}^{3}<1$ ), respectively. We note that in an external Compton scenario ever harder spectra, $F_{\nu} \propto \nu$, can arise.

For illustration, the above is applied to the blazar 1ES $0229+200$ as shown in Fig.1. As it turns out, Maxwell-like distributions can successfully interpret the very hard (de-absorbed) VHE data even in the case of a high level EBL. Note that these functions are steady-state solutions that already take synchrotron losses into account, so that there is no need to invoke very small values for the magnetic field. 


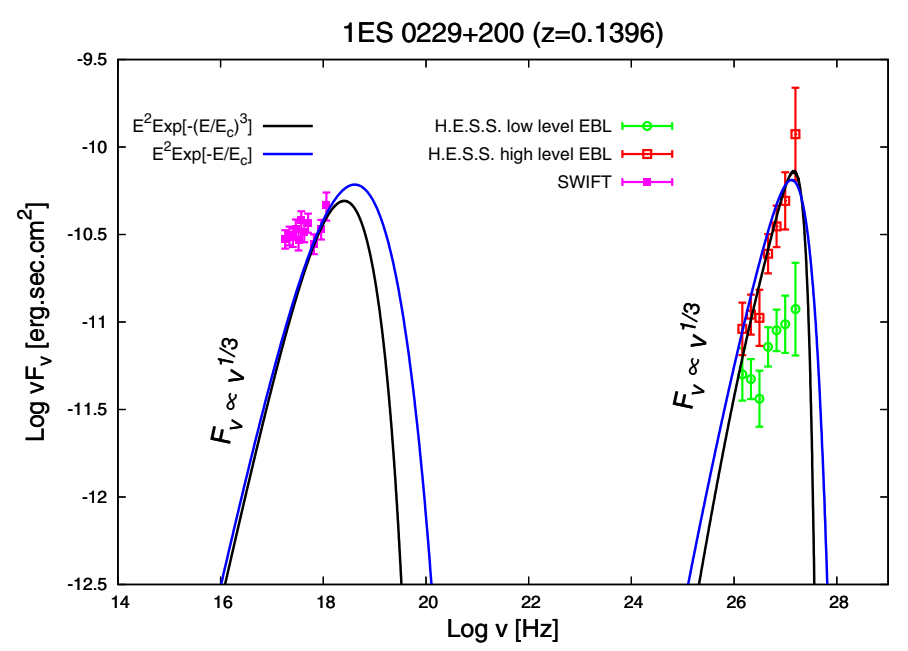

Fig. 1. The blazar 1ES 0229+200 detected at VHE with hard intrinsic spectrum (see Ref. 4). The SED is modeled within an SSC approach using Maxwellian-type electron distributions.

Blue line: Relativistic Maxwellian distribution $n(\gamma)=A \gamma^{2} \exp \left(-\frac{\gamma}{\gamma_{c}}\right)$ with parameters $\gamma_{c}=$ $1.5 \times 10^{5}, B=0.07 \mathrm{G}, A=3 \times 10^{-14} \mathrm{~cm}^{-3}, R=2 \times 10^{14} \mathrm{~cm}$ and $\delta=33$.

Black line: Relativistic Maxwellian distribution $n(\gamma)=A \gamma^{2} \exp \left(-\frac{\gamma}{\gamma_{c}}\right)^{3}$ with parameters $\gamma_{c}=$ $5.3 \times 10^{5}, B=0.06 \mathrm{G}, A=4 \times 10^{-15} \mathrm{~cm}^{-3}, R=2 \times 10^{14} \mathrm{~cm}$ and $\delta=33$.

Data points shown in the figure are from Ref. 8, where the intrinsic (de-absorbed) source spectrum has been derived based on the EBL model of Ref. 9 with (i) EBL level as in their original paper ("low level EBL") and (ii) (maximum) EBL level scaled up by a factor of 1.6 ("high level EBL").Figure taken from Ref. 17.

\section{Broader Emission Spectra and the Case of the Mkn 501 Flare in 2009}

As shown above, relativistic Maxwellian-type distributions can generate hard spectra in the $\mathrm{TeV}$ range. Here we discuss the potential of such distributions to also account for broader and softer emission spectra. This can be illustrated by considering not only a "one-zone" (single component) model, but multiple radiating regions $(N \text { "blobs" })^{18}$. Such a situation could arise, for example, if the flow itself emerges with some internal structure (e.g., shear), or if sub-structures are induced by an external event (e.g., Ref. 21).

The combination of the above electron distributions then leads to power lawlike particle distributions if the temperatures of the different components do not differ significantly and if the components contribute equally to the overall spectra. In the simplest case where all "blobs" are assumed to have the same parameters (e.g., size, magnetic field, total energy) except for their temperatures, the resultant power-law index approaches 2 , i.e., the overall electron distribution approximately follows $d N_{e} / d E \propto E^{-2}$. Steeper spectra could arise if, e.g., the low temperature components dominate, whereas harder spectra may occur if more energy is contained in the high-temperature blobs. Once a single component becomes dominant in the 
overall emission, as naturally expected for a flaring stage, hard spectral features can arise. This is more evident in the Compton part of the spectrum as (in the Thomson regime) the separation of the VHE peaks scales as $\sim \gamma_{c}^{4}$ and is greater than in the synchrotron case $\left(\sim \gamma_{c}^{2}\right)$. The energetics of such a leading component, which is responsible for an observed flare, could change for different reasons. The total (intrinsic) energy offered to the accelerated particles or/and the temperature of the distribution could increase, for example, due to changes in the bulk flow properties or due to an increased finite injection of seed particles that undergo stochastic acceleration. Another possibility concerns an increase of the Doppler factor. Already a slight change of the line-of-sight angle to the observer during the propagation of a blob, could lead to the observation of a hard flare without an accompanying change of the intrinsic energetics of the components.

The aforementioned possibilities can be applied to understand the high-energy flare of Mkn 501 observed in 2009. To illustrate this, we consider four emitting regions $(N=4)$ in which electrons radiate synchrotron photons and up-scatter them to the VHE regime. For the "low state" we assume that the total energy of each component drops as the temperature increases in order to account for the observed, steeper than $\Gamma=1.5 \mathrm{VHE}$ spectrum. For the flaring state the normalization of the two components with the highest temperatures is increased by a factor of $\sim 2$. (We note that both the light-travel time $t_{l} \sim 2 R /(\delta c) \leq 0.1 \mathrm{~d}$ and the

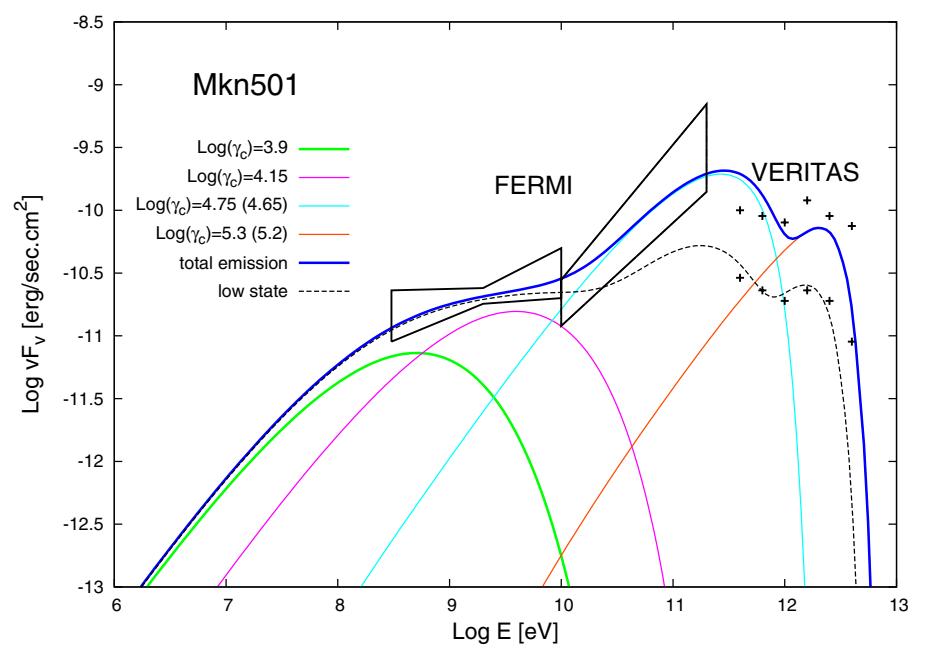

Fig. 2. Resultant SSC emission from the sum of different $(N=4)$ blobs for the "low" (dashedline) and the "flaring state" (blue line) of Mkn 501. The total energy given to the particles scales as $E_{i} \propto \gamma_{c_{i}}^{-1 / 4}$ and for the low state the temperatures are $\log \left(\gamma_{c}\right)=3.9,4.15,4.65$ and 5.2 , respectively. Parameters kept constant are magnetic field $B=0.1 \mathrm{G}$, blob radius $R=10^{14} \mathrm{~cm}$, cut-off index $b_{1}=3$ and Doppler factor $\delta=30$. For the flaring state, the two blobs with highest temperatures are assumed to be enhanced by a factor of $\sim 2$ with their temperatures slightly increased (to $10^{4.75}$ and $10^{5.3}$, respectively). Below $10 \mathrm{GeV}$, the flux is almost constant with respect to the low state. For data points, see Ref. 5 and Ref. 6. Figure taken from Ref. 18. 
observed synchrotron cooling time scale $t_{s} \sim 0.3\left(10^{5} / \gamma_{c}\right)(0.1 \mathrm{G} / \mathrm{B})^{2}(30 / \delta) \mathrm{d}$ are much smaller than the observed duration of the flare. In principle, shorter flares down to timescales of $t_{s}$ could be accommodated). This leads to a hard high-energy flare above $10 \mathrm{GeV}$. Below $10 \mathrm{GeV}$ the emission stays almost constant. Other parameters are kept the same for all blobs (i.e., magnetic field $B=0.1 \mathrm{G}$, blob radius $R=10^{14} \mathrm{~cm}$ and Doppler factor $\left.\delta=30\right)$. For this set of parameters, the synchrotron flux is lower than the one observed, so that the X-ray emission would have to come from a different (additional) part of the jet. This is consistent with the fact that little flux variation has been observed in X-rays.

\section{Conclusions}

The hard intrinsic VHE spectra of some BL Lac objects have been difficult to interpret within standard leptonic models. As shown above, however, narrow energetic electron distributions and relativistic Maxwellian-like particle spectra that are expected in stochastic acceleration scenarios, are cable to reproduce hard TeV photon spectra. A combination of different components may as well lead to broader and softer emission spectra. In the latter case, hard spectral features can become apparent during flaring states, as illustrated here for the 2009 flare of Mkn 501, once one or a few components become dominant over the background.

\section{References}

1. R. J. Gould and G. P. Schreder, Physical Review 155, 1408 (1967).

2. J. R. Primack, et al., in Proc. of the 25th Texas Symposium on Relativistic Astrophysics, eds. F. A. Aharonian, W. Hofmann and F.M. Rieger, AIP 1381 (2011), pp. 72-83.

3. F. Aharonian et al., Nature 440, 1018 (2006).

4. F. Aharonian et al., Astron. Astrophys. 475, L9 (2007).

5. A. A. Abdo et al., Astrophys. J. 727, 129 (2011).

6. A. Neronov, D. Semikoz, and A. M. Taylor, submitted [arXiv:1104.2801].

7. F. A. Aharonian, D. Khangulyan and L. Costamante, Mon. Not. R. Astron. Soc. 387, 1206 (2008).

8. O. Zacharopoulou et al., Astrophys. J. 738, 157 (2011).

9. A. Franceschini, G. Rodigliero and M. Vaccari, Astron. Astrophys. 487, 837 (2008).

10. M. Böttcher, C. D. Dermer and J. D. Finke, Astrophys. J. Lett. 679, L9 (2008).

11. F. W. Stecker, M. G. Baring and E. J. Summerlin, Astrophys. J. Lett. 667, L29 (2007).

12. F. A. Aharonian et al., Astron. Astrophys. 384, 834 (2002).

13. W. Essey et al., Astrophys. J. 731, 51E (2011).

14. A. De Angelis et al., Mon. Not. R. Astron. Soc. 394, L21 (2009).

15. T. Kifune, Astrophys. J. Lett. 518, L21 (1999).

16. K. Katarzyński et al., Mon. Not. R. Astron. Soc. 368, L52 (2007).

17. E. Lefa, F. M. Rieger, and F. A. Aharonian, Astrophys. J. 740, 64 (2011).

18. E. Lefa, F. A. Aharonian and F. M. Rieger, Astrophys. J. Lett. (2011), accepted [arXiv: 1108.4568].

19. R. Schlickeiser, Astron. Astrophys. 143, 431 (1985).

20. F. A. Aharonian et al., Astron. Astrophys. 162, L1 (1986).

21. M.V. Barkov, F.A. Aharonian and V. Bosch-Ramon, Astrophys. J. 724, 1517 (2010). 